\title{
CYP1A1 is a target of miR-892a-mediated post-transcriptional repression
}

\author{
YEONG MIN CHOI $^{*}$, SUNGKWAN AN $^{1,2^{*}}$, EUN-MEE LEE $^{1}$, KARAM KIM $^{1}$, SUNG JIN CHOI $^{1}$, \\ JU SUB KIM ${ }^{3}$, HYUN HEE JANG ${ }^{4}$, IN-SOOK AN ${ }^{1,2}$ and SEUNGHEE BAE ${ }^{1}$ \\ ${ }^{1}$ Functional Genoproteome Research Centre and LIFEnGENE Inc.; ${ }^{2}$ Aesthetics and Cosmetology Research Institute, \\ Konkuk University, Seoul 143-701; ${ }^{3}$ Department of Cosmetology, Sangji Youngseo College, Wonju-si, Gwangwon-do \\ 220-713; ${ }^{4}$ School of Cosmetology, Kyungbok University, Pocheon-si, Gyeonggi-do 487-717, Republic of Korea
}

Received December 27, 2011; Accepted March 2, 2012

DOI: $10.3892 /$ ijo.2012.1418

\begin{abstract}
Cytochrome P450 1A1 (CYP1A1) is a member of the cytochrome p450 enzyme family, which is involved in the metabolisms of carcinogenic metabolites, such as benzo(a) pyrene. In this study, we identified miR-892a as a negative regulator of CYP1A1 expression. Luciferase assays revealed a sequence in the 3'-untranslated region of CYP1A1 that displayed a perfect match with miR-892a, and revealed that this sequence was a specific miR-892a target site. The overexpression of miR-892a inhibited the expression of the CYP1A1 protein, and the miR-892a antagonist increased CYP1A1 expression. Of note, benzo(a)pyrene, a major inducer of CYP1A1 transcription, decreased the expression of miR-892a. Moreover, the miR-892a-induced CYP1A1 repression inhibited the benzo(a) pyrene-mediated decrease in cell viability. These data provide insight into the CYP1A1 regulatory network.
\end{abstract}

\section{Introduction}

Cytochrome P450 1A1 (CYP1A1) is a key carcinogen-metabolizing enzyme that catalyzes the hydroxylation of environmental carcinogens including benzo(a)pyrene (BaP) (1). After cells are exposed to $\mathrm{BaP}$-containing pollutants, $\mathrm{BaP}$ binds to and activates the aryl hydrocarbon receptor (AhR), which subsequently translocates to the nucleus (2). The activated AhR interacts with the aryl hydrocarbon nuclear translocator and induces the upregulation of CYP1A1 expression (3). The upregulated CYP1A1 converts BaP into a metabolite that can form DNA adducts. Moreover, high CYP1A1 inducibility phenotypes

Correspondence to: Dr Seunghee Bae, Functional Genoproteome Research Centre, Konkuk University, 1 Hwayang-dong, Gwangjin-gu, Seoul 143-701, Republic of Korea

E-mail: sbae@konkuk.ac.kr

*Contributed equally

Key words: cytochrome P450 1A1, benzo(a)pyrene, microRNA, miR-892a, cell viability and the MspI polymorphism are suspected to correlate with increased lung cancer frequency.

Although AhR is a major regulator of CYP1A1 gene expression, other regulatory proteins have been reported to be involved in CYP1A1 induction. For instance, peroxisome proliferatoractivated receptor $\alpha(\operatorname{PPAR} \alpha)$, hepatocyte nuclear factor 4 (HNF4), and its co-activator, PPAR $\gamma$ coactivator $1 \alpha$ (PGC1 $\alpha$ ), have been found to induce CYP1A1 transcription. Furthermore, the transcription factor OCT-1 inhibits CYP1A1 expression. However, these factors merely control CYP1A1 transcription.

MicroRNAs (miRNAs) are small, non-coding RNAs that function as post-transcriptional regulators to inhibit mRNA translation (4-6). The presence of miRNAs has been described in heterogenic cell processes, such as differentiation, apoptosis, hematopoiesis, fat metabolism and limb morphogenesis (7-10). A number of miRNAs have been identified and have provided important information as regards drug metabolism. miR-27b is a specific negative regulator of CYP1B1 that is known to be involved in drug metabolism and tumorigenesis $(11,12)$. miR-27a and miR-451, which are upregulated in multidrug-resistant cancer cell lines, induce the overexpression of the drug resistance-related protein, P-glycoprotein, in multidrug-resistant cell lines (13). Although many studies have demonstrated the importance of miRNAs in the regulation of drug-metabolizing enzymes and drug transporters, the negative regulation of CYP1A1 expression at the post-transcriptional level remains unclear. In this study, we demonstrate that the translational repression of CYP1A1 mRNA expression is mediated by miR-892a.

\section{Materials and methods}

RNA oligonucleotides and chemicals. The miRNA mimic for miR-892a, antagonist (antago)-mir-892a, CYP1A1 siRNA, and a negative control were purchased from Qiagen (Hilden, Germany). All miRNA mimics and siRNAs were re-suspended in nuclease-free water (USB, Cleveland, $\mathrm{OH}, \mathrm{USA}$ ) to a final concentration of $20 \mu \mathrm{M}$. BaP was purchased from Sigma (St. Louis, MO, USA).

Cell lines and transfection. The human breast adenocarcinoma cell line, MCF7, was obtained from the American Type Culture 
Collection (Manassas, VA, USA). The cells were cultured in MEM supplemented with $10 \%$ fetal bovine serum, $100 \mathrm{U} / \mathrm{ml}$ penicillin, and $100 \mu \mathrm{g} / \mathrm{ml}$ streptomycin and maintained at $37^{\circ} \mathrm{C}$ in a $5 \% \mathrm{CO}_{2}$ incubator. Transfection was performed using Lipofectamine RNAiMAX (Invitrogen, Carlsbad, CA, USA) for $5 \mathrm{~h}$ in serum-free medium. After changing the medium, the cells were incubated for 24-48 $\mathrm{h}$ and harvested.

Quantitative real-time PCR analysis. The cDNAs were synthesized from total RNAs by using the miScript Reverse Transcription kit (Qiagen) according to the manufacturer's instructions. The primer for human precursor miR-892a (pre-miR-892a) was 5'-CACUGUGUCCUUUCUGCGUAG-3'. The forward and reverse primers for human U6 snRNA were 5'-CGCTTCGGCAGCACATATACTAA-3' and 5'-TATGGA ACGCTTCACGAATTTGC-3', respectively. The cycling protocol for human pre-miR-892a was as follows: initial denaturation at $95^{\circ} \mathrm{C}$ for $5 \mathrm{~min}$, followed by 45 cycles of $95^{\circ} \mathrm{C}$ for $15 \mathrm{sec}, 63^{\circ} \mathrm{C}$ for $20 \mathrm{sec}$, and $72^{\circ} \mathrm{C}$ for $30 \mathrm{sec}$. The PCR protocol for human U6 snRNA was as follows: initial denaturation at $95^{\circ} \mathrm{C}$ for $30 \mathrm{sec}$, followed by 45 cycles of $94^{\circ} \mathrm{C}$ for $10 \mathrm{sec}$ (denaturation) and $62^{\circ} \mathrm{C}$ for $20 \mathrm{sec}$ (annealing and extension). PCR was performed using the miScript SYBRGreen PCR kit (Qiagen) with Line-Gene K software (Bioer Technology Co. Ltd., Hangzhou, China).

Cell viability assay. Cell viability was assessed using the MTS assay according to the manufacturer's instructions (Sigma). The results are graphically represented as the measured cell viability ratio normalized to the control.

Western blot analysis. Western blot analysis was performed as described previously (14). A primary antibody specific for CYP1A1 was obtained from Santa Cruz Biotechnology (Santa Cruz, CA, USA). An anti- $\beta$-actin antibody was purchased from Sigma-Aldrich.

Construction of 3'-UTR reporter plasmids and luciferase assay. To construct luciferase reporter plasmids, various target fragments were ligated into the $X b a \mathrm{I}$ site downstream of the luciferase gene in the pGL3-promoter vector. The sequence of the miR-892a recognition element (MRE892a) spanned from 2021 to +2080 of the human CYP1A1 gene (5'-GAACUAGG CUGCAAUCUGACCCUAUGGGUCUAGGACACAGUUCCU GGGAA-3'). A fragment containing the perfect matching sequence of the mature miR-892a, 5'-CUAUGGGUCUAGG $A C A C A G U-3$ ' (the matching sequence of miR-892a is italicized), was cloned into the pGL3 promoter vector (pGL3-MRE892a). The region $(+1661$ to +2609$)$ in the human CYP1A1 gene containing MRE892a was amplified by PCR using the following primers adapted to the $\mathrm{XbaI}$ site: 5'-GCTCTAGAGTGCTT GAGAGCCCTGAG-3' and 5'-GCTCTAGA CTGAACAATAT CTTTATTTAGC-3'. The pGL3 promoter plasmid containing the 3'-UTR of CYP1A1 was termed pGL3-3'UTR. DNA sequencing analyses confirmed the nucleotide sequences of the constructed plasmids. For luciferase assays, various luciferase reporter plasmids (pGL3) were transiently transfected with the precursors for miR-892a, the control, or the antago-miR for miR-892a and the pSV- $\beta$-gal plasmid into the cells. Briefly, 500 ng pGL3 plasmid and 200 ng pSV- $\beta$-gal plasmid were transfected using Lipofectamine plus reagent into the cells. After incubation for $48 \mathrm{~h}$, the cells were re-suspended in Passive Lysis Buffer (Promega Corp., Madison, WI, USA), and then the luciferase activity was measured with a Veritas Luminometer (Turnur Designs, Sunnyvale, CA). The relative luciferase activity was normalized to $\beta$-galactosidase activity and presented as the fold activation with standard deviations. The results are the averages of three independent experiments.

\section{Results}

miR-892 a contains a complementary sequence of the 3'-UTR of human CYPIAl mRNA. To identify human miRNAs that can regulate CYP1A1 expression, we used the target scan program to predict miRNAs and found that miR-892a was highly scored for targeting CYP1A1 in all three algorithms, namely TargetScan, miRanda (MicroCosm), and PicTar (15-17). The predicted target site for miR-892a is encoded by nucleotides 515-535 in the 3'-UTR of CYP1A1 mRNA (Fig. 1). In addition, miR-892a contains a conserved target sequence of the 3 '-UTR of CYP1A1 in humans and other mammalian species, including the chimpanzee. This region was termed MRE892a. From these in silico results illustrating that a cognate miR-892a target region is located in the 3'-UTR of CYP1A1, we hypothesized that miR-892a could modulate the expression of CYP1A1 by targeting MRE892a.

miR-892a regulates the $m R N A$ expression of $C Y P 1 A 1$. First, we determined whether the expression of miR-892a and CYP1A1 is negatively correlated. To investigate this, we used antagomiRNA to reduce the levels of endogenous miR-892a in MCF7 cells. The treatment of the cells with antago-miR-892a reduced miR-892a expression (Fig. 2A) and increased the mRNA and protein expression of CYP1A1 (Fig. 2B). It has previously been reported that CYP1A1 mRNA and protein expression are induced by $\mathrm{BaP}$ via the activation of $\mathrm{AhR}$, which is a known CYP1A1 gene transcription factor. Therefore, we next examined whether the CYP1A1 mRNA expression was affected by miR-892a. As shown in Fig. 2C, the expression levels of CYP1A1 mRNA and protein were dramatically increased after $\mathrm{BaP}$ treatment in the cells; however, this upregulation was markedly inhibited by treatment with miR-892a mimics compared with the inhibition induced by the control and control mimics. These results indicate that miR-892a is an effective post-transcriptional regulator of CYP1A1 mRNA.

3'-UTR of CYPIAl is a target for miR-892a. We then examined whether the negative regulation of CYP1A1 expression by $\mathrm{miR}-892 \mathrm{a}$ is due to a direct interaction between miR-892a and the predicted binding site in the 3'-UTR of CYP1A1. We therefore constructed recombinant luciferase reporter plasmids in which the 3'-UTR (pGL3-3'-UTR) and MRE892a (pGL3MRE892a) of CYP1A1 were cloned downstream of the firefly luciferase reporter gene in pGL3 vectors (Fig. 3A). These reporter plasmids were co-transfected with either the miR-892a mimics or its antago-miR in MCF7 cells, and then luciferase activity was measured after $24 \mathrm{~h}$ after transfection. As shown in Fig. 3B, the overexpression of miR-892a markedly decreased the luciferase activities of pGL3-3'-UTR and pGL3-MRE892a by 30 and $50 \%$, respectively, compared to those of the control. 


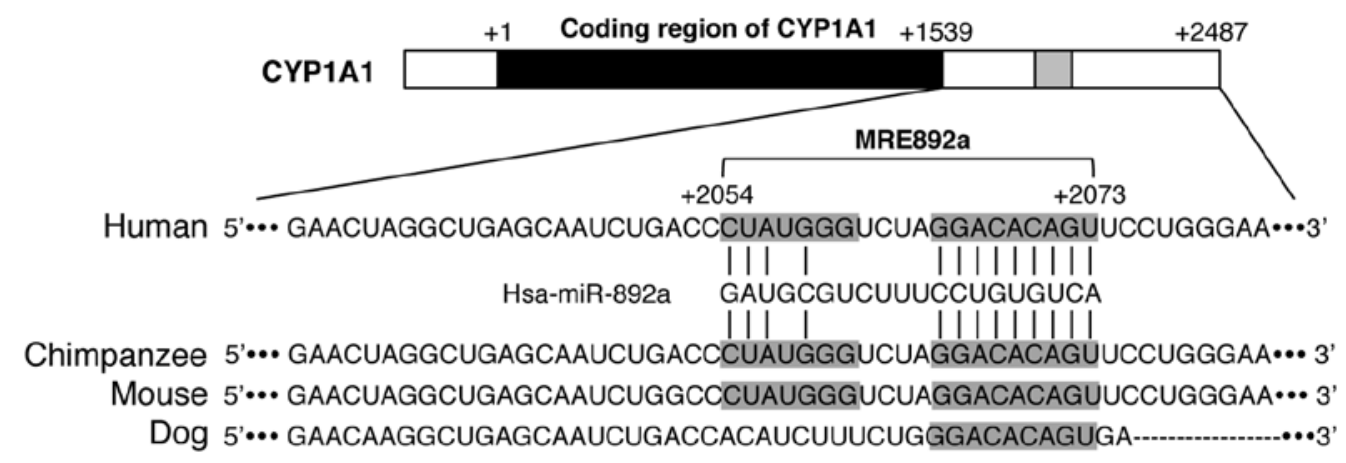

Figure 1. In silico analysis of CYP1A1 mRNAs and the predicted target sequence of miR-892a. As regards numbering, the ATG codon is numbered +1 , and the coding region of human CYP1A1 spans to +1539 (black box). The sequences of the 3'-UTR and MRE892a are located from +1540 to +2487 and from +2054 to +2073 in human CYP1A1 mRNA, respectively. Gray boxes indicate the target-specific sites for miR-892a (MRE892a) in the 3'-UTRs of CYP1A1 mRNAs.

A

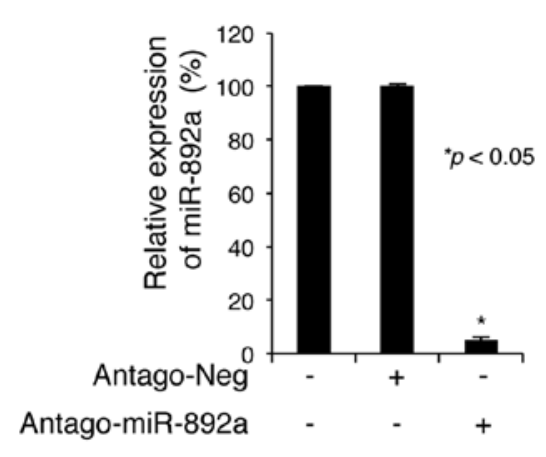

C
B

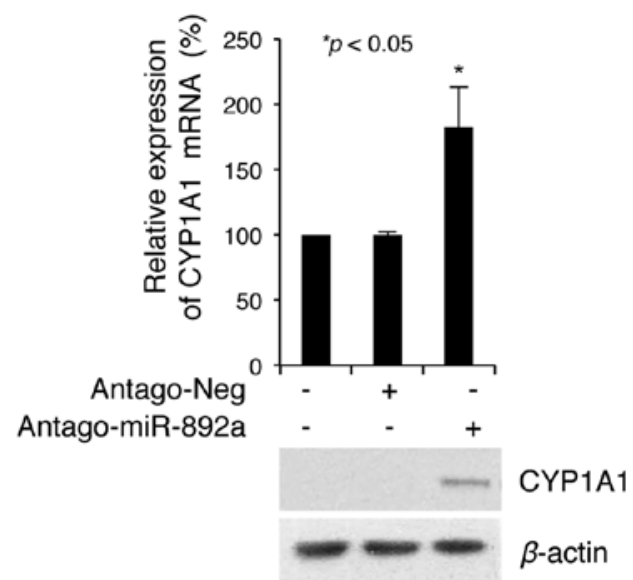

CYP1A1

$\beta$-actin

Figure 2. CYP1A1 expression is regulated by miR-892a. (A) Antago-miR-892a reduced the levels of miR-892a in MCF7 cells. The cells were treated with antago-miR-892a or the negative control $(100 \mathrm{nM})$. The level of miR-892a was determined by real-time PCR. Values are presented as the pre-miR-892a level normalized to the U6 snRNA level. The results are representative of three independent experiments (means \pm SE). Antago-Neg, negative-control antago-miR. (B) Antago-miR-892a upregulated CYP1A1 expression. MCF7 cells were transfected with the indicated miRNAs. The mRNA and protein levels of CYP1A1 were determined by real-time PCR and immunoblotting, respectively. (C) BaP-mediated CYP1A1 induction was inhibited by miR-892a. After transfection with miR-892a and control (100 nM) as indicated, MCF7 cells were treated with $1 \mu \mathrm{M} \mathrm{BaP}$ for $24 \mathrm{~h}$ and then subjected to real-time PCR and immunoblotting with the indicated antibodies. miR-Neg, negative-control miR. "Statistical significance compared with the findings in the control cells for each condition ( $\mathrm{p}<0.05)$.

Inversely, this downregulation was rescued by co-transfection with antago-miR-892a, indicating that miR-892a negatively regulates CYP1A1 expression post-transcriptionally by directly recognizing MRE892a in the 3'-UTR of CYP1A1.
BaP decreases miR-892a expression. As the upregulation of CYP1A1 expression is highly induced by $\mathrm{BaP}$ treatment (3), we examined whether BaP could affect the expression level of CYP1A1 by regulating miR-892a expression. To test this hypoth- 
A

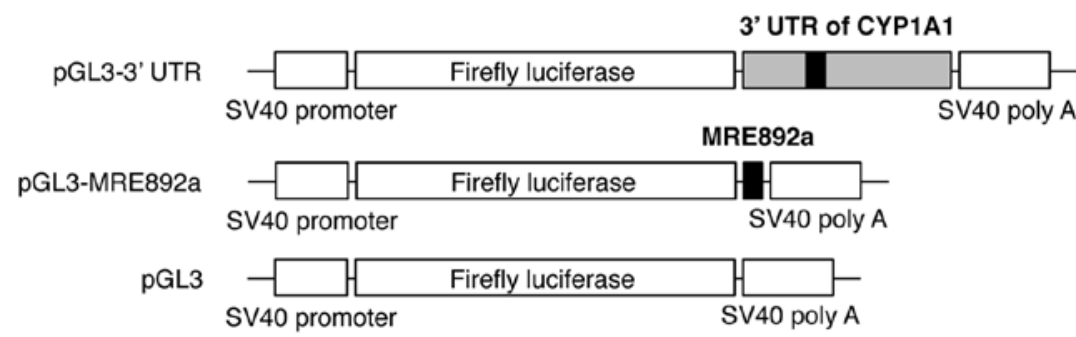

B

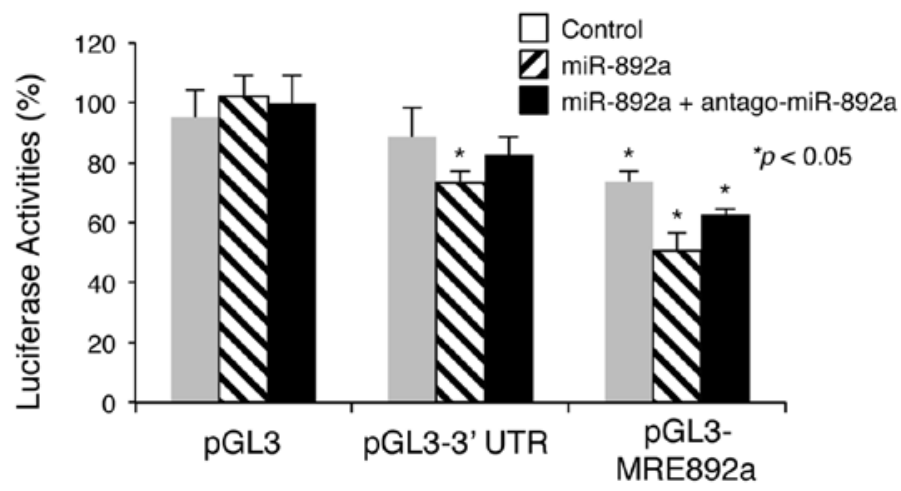

Figure 3. CYP1A1 is a target of miR-892a. (A) A series of luciferase reporter constructs containing the 3'-UTR and MRE892a of CYP1A1 genes. The 3'-UTR and MRE892a were inserted in the downstream coding region of the luciferase gene. The gray box indicates the 3'-UTR of CYP1A1. The black box indicates the specific target site for miR-892a (MRE892a) in the 3'-UTR. (B) miR-892a directly recognizes MRE892a in the 3'-UTR of CYP1A1. MCF7 cells were co-transfected with a series of luciferase reporter plasmids, miR-892a mimics, and antago-miR-892a as indicated. After $24 \mathrm{~h}$ of incubation, cells were lysed, and the reporter luciferase activities were determined. All of the experiments were performed twice in triplicate $(n=6)$. *Statistical significance compared with the findings in the control cells for each condition $(\mathrm{p}<0.05)$.

esis, we first examined the expression levels of miR-892a upon stimulation with $\mathrm{BaP}$ in $\mathrm{MCF} 7$ cells. Interestingly, $\mathrm{BaP}$ greatly decreased miR-892a expression in a concentration-dependent manner (Fig. 4A). This result led to us examine whether BaP could decrease not only the expression level of miR-892a, but also the activity of this miRNA activity by blocking the translation of the CYP1A1 transcript. To test this hypothesis, we used recombinant luciferase reporter plasmids, such as pGL3 control, pGL3-3'-UTR and pGL3-MRE892a (see Materials and methods) and co-transfected these plasmids with antagomiR-892a into BaP-stimulated MCF7 cells. We found that the luciferase activities of pGL3-3'-UTR and pGL3-MRE892a were decreased by 20 and $30 \%$, respectively, compared to those in the control pGL3-transfected MCF7 cells (Fig. 4B). However, this downregulation was completely blocked by $\mathrm{BaP}$ stimulation, suggesting that $\mathrm{BaP}$ negatively regulates miR-892a expression and activity (Fig. 4B). Similarly, antago-miR-892a perfectly restored the downregulated luciferase activities in pGL3-3'-UTR- and pGL3-MRE892a-transfected cells (Fig. 4B). These results indicate that the regulatory mechanism between miR-892a and its target CTP1A1 is controlled by BaP stimulation.

miR-892a inhibits BaP-induced loss of cell proliferation. As the CYP1A1 upregulation, which is induced by BaP stimulation, decreases cell proliferation (18), we investigated the effect of miR-892a on proliferation in BaP-stimulated cells. First, we confirmed that BaP decreased MCF7 cell viability and induced CYP1A1 expression in a concentration-dependent manner
(Fig. 5A). The MCF7 cells were then transfected with CYP1A1 target-specific siRNA. Following $24 \mathrm{~h}$ of transfection, cells were treated with $40 \mu \mathrm{M}$ BaP for $24 \mathrm{~h}$. The siRNA completely blocked CYP1A1 expression (Fig. 5B, bottom panel) and inhibited the BaP-induced loss of cell viability compared to the findings in control and negative control siRNA-transfected cells (Fig. 5B, top panel). Similarly, miR-892a mimics also restored the BaP-induced loss of cell viability (Fig. 5C). These results suggest that miR-892a negatively regulates CYP1A1 function by inhibiting cell growth suppression in the presence of $\mathrm{BaP}$.

\section{Discussion}

The present study demonstrates that miR-892a is a direct transcriptional target of CYP1A1 that negatively regulates BaP-induced cell growth suppression by inhibiting CY1A1 expression. Our in silico analysis revealed that miR-892a has a high-affinity binding site in the 3'-UTR of CYP1A1. In MCF7 cells, antago-miR-892a upregulated CYP1A1 mRNA and protein expression. Conversely, the miR-892a mimics decreased the expression level of CYP1A1 in MCF7 cells. Furthermore, luciferase reporter assays revealed that miR-892a can directly target the MRE892a-containing region in the 3'-UTR of CYP1A1. Of note, both the expression level and the translation-repressing activity of miR-892a were decreased by $\mathrm{BaP}$ stimulation, which is a well-known inducer of CYP1A1 expression in cells. In addition, the BaP-mediated loss of cell viability was blocked through the repression of CYP1A1 expres- 
A

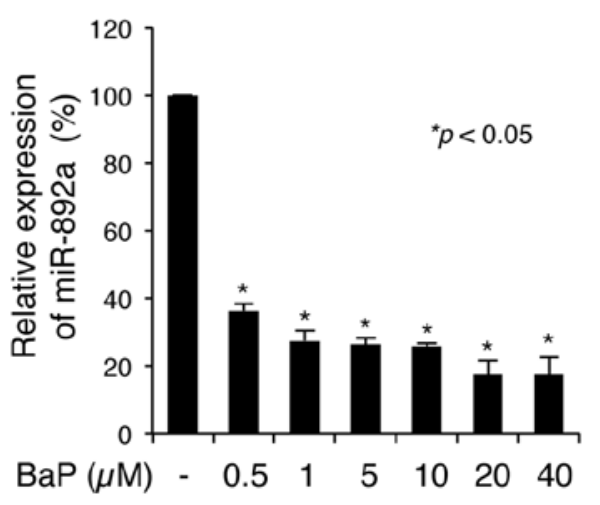

B

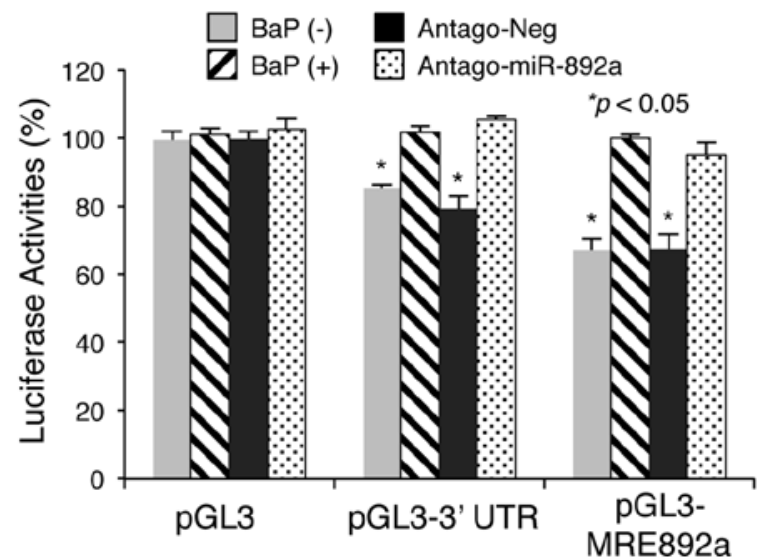

Figure 4. BaP regulates the expression of miR-892a. (A) BaP reduced miR-892a expression in a concentration-dependent manner. MCF7 cells were treated with $\mathrm{BaP}$ at the indicated concentrations. After $24 \mathrm{~h}$ of incubation, the levels of miR-892a were determined by real-time PCR. The results are representative of three independent experiments (means \pm SE). (B) BaP reduced miR-892a activity for targeting the 3'-UTR of CYP1A1. A series of luciferase reporter plasmids, miR892a mimics, and antago-miR-892a were introduced into MCF7 cells, and then the luciferase activities were determined. "Statistical significance compared with the findings in the control cells for each condition $(\mathrm{p}<0.05)$.

A

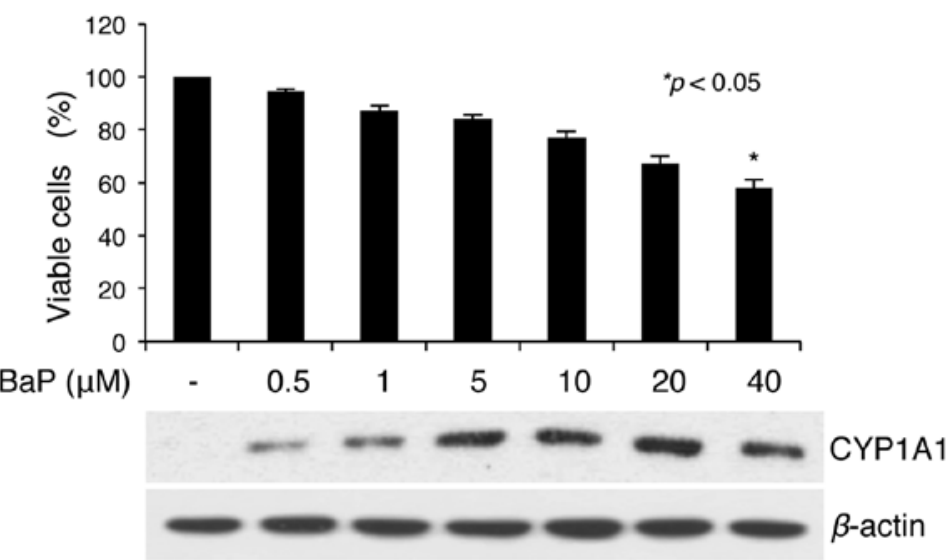

B

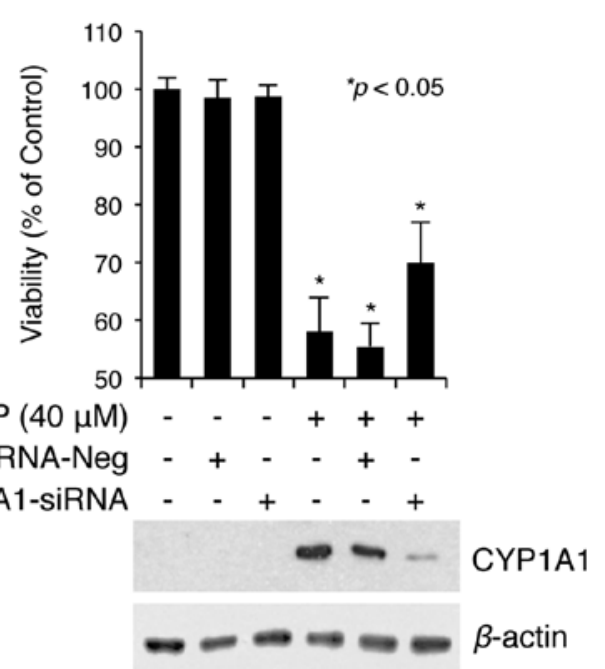

C

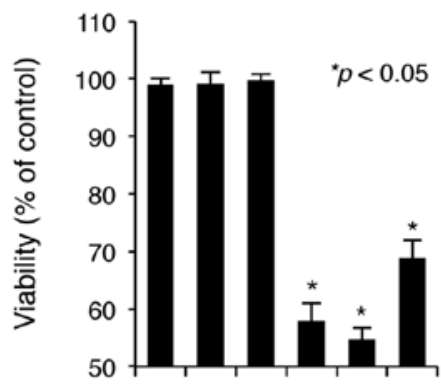

$\operatorname{BaP}(40 \mu \mathrm{M})-\quad-+++$

miR-Neg - +--+-

miR-892a - - + - +

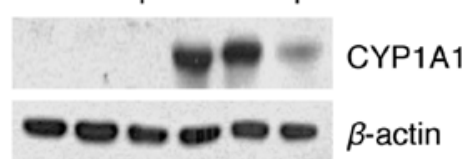

Figure 5. miR-892a suppresses the cell growth-inhibitory function of BaP. (A) BaP inhibited MCF7 cell proliferation in a concentration-dependent manner. MCF7 cells were treated with $\mathrm{BaP}$ as indicated, and then the cell viabilities were determined by MTT assay. BaP-mediated CYP1A1 induction was confirmed by immunoblotting with the indicated antibodies. (B and C) Growth inhibitory function of BaP was inhibited by CYP1A1 depletion by (B) its specific siRNA and (C) miR-892a. After transfection of MCF7 cells with (B) CYP1A1 siRNA or (C) miR-892a mimics, $40 \mu \mathrm{M}$ BaP was added for $24 \mathrm{~h}$ and then the cell viabilities were determined by MTT assay. The silencing induced by siRNA and miRNA was confirmed by immunoblotting with the indicated antibodies. The results are representative of three independent experiments (means $\pm \mathrm{SE}$ ). *Statistical significance compared with the findings in the control cells at each condition ( $\mathrm{p}<0.05$ ). 
sion by miR-892a overexpression. Overall, our data indicate that miR-892a is a novel regulator of BaP-induced CYP1A1 expression by directly repressing CYP1A1 expression, inducing $\mathrm{BaP} /$ CYP1A1-mediated cell growth suppression.

Recent studies have highlighted the importance of 3'-UTRs in gene regulation, especially via miRNA-mediated interference or silencing of mRNA (19). Many types of CYP mRNAs have different sequences in different human populations; therefore, miRNA and siRNA play an important role in this regulation. For example, miR-27b has a near-perfect matching sequence in the 3'-UTR of CYP1B1, through which it directly suppresses CYP1B1 gene expression. In particular, the expression levels of miR-27b and CYP1B1 protein in cancerous and adjacent non-cancerous breast tissues from patients are significantly and inversely correlated $(11,20)$. CYP17 is critical in androgen biosynthesis, and CYP17 mRNA expression has been proven to be augmented in thecal cells from a patient with polycystic ovary syndrome. siRNA-mediated CYP17 mRNA depletion can reduce steroid synthesis in cells (21). siRNA duplexes targeting CYP4B1 reduced the expression of this gene by $60 \%$, and 12-HETrE levels were barely detected in corneal homogenates from eyes. These results strongly implicate corneal CYP4B1 as a component of the inflammatory and neovascular cascade initiated by injury (22). Recently, analysis of genetic variation revealed a region of the CYP1A1 locus having decreased nucleotide diversity in the pooled populations, possibly due to a selective sweep (23). Of note, the analysis of the 3'-UTR of the CYP1A1 gene revealed that most single nucleotide polymorphisms (SNPs) are concentrated in the region (23). In addition, an in silico study identified different miRNA-binding patterns in the region when mutations were present compared to when mutations were absent (23). These data suggest that SNPs affected by selective sweeping, which are located in the CYP1A1 3'-UTR are worthy of further investigation. Although the miR-892a expression levels in tissues or populations have not yet been researched, our findings, for the first time, suggest that miR-892a can regulate the CYP1A1 pathway activated by pollutants such as BaP.

Although AhR mainly induces BaP-mediated CYP1A1 expression, our findings indicate that this novel pathway of BaP-mediated miR-892a suppression can also induce CYP1A1 expression. There are a number of reports demonstrating AhR-independent CYP1A1 gene expression. For example, dibenso( $\alpha$ )pyrene has been shown to induce CYP1A1 mRNA expression in AhR knockout mice (24). In addition, 3-methylcholanthrene has been shown to increase CYP1A1 protein levels in AhR knockout mice (25).

Although the accurate mechanisms of BaP-dependent miR-892a downregulation are largely uncovered, the aforementioned reports and our findings indicate that an AhR-independent pathway provides a possible future investigation topic for CYP1A1-related fields.

\section{Acknowledgements}

We are grateful to all the other members of our research group for their support and advice regarding this study. The present study was supported by grants from the Ministry of Education, Science and Technology (grants 20110028646 and 20110018427 to S.A.) of the Republic of Korea.

\section{References}

1. McManus ME, Burgess WM, Veronese ME, Huggett A, Quattrochi LC and Tukey RH: Metabolism of 2-acetylaminofluorene and benzo(a)pyrene and activation of food-derived heterocyclic amine mutagens by human cytochromes P-450. Cancer Res 50: 3367-3376, 1990.

2. Nakatsuru Y, Wakabayashi K, Fujii-Kuriyama Y, Ishikawa T, Kusama K and Ide F: Dibenzo[A,L]pyrene-induced genotoxic and carcinogenic responses are dramatically suppressed in aryl hydrocarbon receptor-deficient mice. Int J Cancer 112: 179-183, 2004.

3. Rowlands JC and Gustafsson JA: Aryl hydrocarbon receptormediated signal transduction. Crit Rev Toxicol 27: 109-134, 1997.

4. Couzin J: Nobel Prize in Physiology or Medicine. Method to silence genes earns loud praise. Science 314: 34, 2006.

5. Ambros V: The functions of animal microRNAs. Nature 431: 350-355, 2004

6. Ambros V and Chen X: The regulation of genes and genomes by small RNAs. Development 134: 1635-1641, 2007.

7. Brennecke J, Hipfner DR, Stark A, Russell RB and Cohen SM: bantam encodes a developmentally regulated microRNA that controls cell proliferation and regulates the proapoptotic gene hid in Drosophila. Cell 113: 25-36, 2003.

8. Chen CZ, Li L, Lodish HF and Bartel DP: MicroRNAs modulate hematopoietic lineage differentiation. Science 303: 83-86, 2004.

9. Cuellar TL and McManus MT: MicroRNAs and endocrine biology. J Endocrinol 187: 327-332, 2005.

10. Harfe BD, McManus MT, Mansfield JH, Hornstein E and Tabin CJ: The RNaseIII enzyme Dicer is required for morphogenesis but not patterning of the vertebrate limb. Proc Natl Acad Sci USA 102: 10898-10903, 2005.

11. Tsuchiya Y, Nakajima M, Takagi S, Taniya T and Yokoi T: MicroRNA regulates the expression of human cytochrome P450 1B1. Cancer Res 66: 9090-9098, 2006.

12. Roos PH and Bolt HM: Cytochrome P450 interactions in human cancers: new aspects considering CYP1B1. Expert Opin Drug Metab Toxicol 1: 187-202, 2005.

13. Zhu H, Wu H, Liu X, et al: Role of MicroRNA miR-27a and miR-451 in the regulation of MDR1/P-glycoprotein expression in human cancer cells. Biochem Pharmacol 76: 582-588, 2008.

14. Kim SY, Bae S, Choi KH and An S: Hydrogen peroxide controls Akt activity via ubiquitination/degradation pathways. Oncol Rep 26: 1561-1566, 2011.

15. Lewis BP, Shih IH, Jones-Rhoades MW, Bartel DP and Burge CB: Prediction of mammalian microRNA targets. Cell 115: 787-798, 2003.

16. Enright AJ, John B, Gaul U, Tuschl T, Sander C and Marks DS: MicroRNA targets in Drosophila. Genome Biol 5: R1, 2003.

17. Krek A, Grun D, Poy MN, et al: Combinatorial microRNA target predictions. Nat Genet 37: 495-500, 2005.

18. Zhang L and Shiverick KT: Benzo(a)pyrene, but not 2,3,7,8-tetrachlorodibenzo-p-dioxin, alters cell proliferation and c-myc and growth factor expression in human placental choriocarcinoma JEG-3 cells. Biochem Biophys Res Commun 231: 117-120, 1997.

19. Chua JH, Armugam A and Jeyaseelan K: MicroRNAs: biogenesis, function and applications. Curr Opin Mol Ther 11: 189-199, 2009.

20. McFadyen MC, Breeman S, Payne S, et al: Immunohistochemical localization of cytochrome P450 CYP1B1 in breast cancer with monoclonal antibodies specific for CYP1B1. J Histochem Cytochem 47: 1457-1464, 1999.

21. Liu Y, Yao ZX and Papadopoulos V: Cytochrome P450 17alpha hydroxylase/17,20 lyase (CYP17) function in cholesterol biosynthesis: identification of squalene monooxygenase (epoxidase) activity associated with CYP17 in Leydig cells. Mol Endocrinol 19: 1918-1931, 2005.

22. Seta F, Patil K, Bellner L, et al: Inhibition of VEGF expression and corneal neovascularization by siRNA targeting cytochrome P450 4B1. Prostaglandins Other Lipid Mediat 84: 116-127, 2007.

23. Jorge-Nebert LF, Jiang Z, Chakraborty R, et al: Analysis of human CYP1A1 and CYP1A2 genes and their shared bidirectional promoter in eight world populations. Hum Mutat 31: 27-40, 2010.

24. Kerzee JK and Ramos KS: Constitutive and inducible expression of Cypla1 and Cyplb1 in vascular smooth muscle cells: role of the Ahr bHLH/PAS transcription factor. Circ Res 89: 573-582, 2001.

25. Kondraganti SR, Fernandez-Salguero P, Gonzalez FJ, Ramos KS, Jiang W and Moorthy B: Polycyclic aromatic hydrocarboninducible DNA adducts: evidence by 32P-postlabeling and use of knockout mice for Ah receptor-independent mechanisms of metabolic activation in vivo. Int J Cancer 103: 5-11, 2003. 